\title{
Die Gesundheitsförderer
}

\author{
Erhard Taverna
}

Art. 19 des Bundesgesetzes über die Krankenversicherung (KVG) bildet die gesetzliche Grundlage für die Aufgaben von «Gesundheitsförderung Schweiz». 90 Projekte und 3 Schwerpunktprogramme werden mit 20 Rappen pro Krankenversicherte/n monatlich finanziert. Der Präsident des Stiftungsrates empfiehlt ein Nein zur Volksabstimmung über das Steuerpaket. Er und eine grosse Zahl kommunaler und kantonaler Akteure beklagen, dass der radikale Sparkurs von Bundesrat und Parlament zum sicheren «Grounding» von Prävention und Gesundheitsförderung führen. Auf den Vorschlag aus dem Aargau für eine nationale Fettsteuer ist das Parlament nicht eingegangen, auf sein Gesuch um 5 Rappen zusätzlich auch nicht.

\section{Die Betriebe}

Während der Staat seine Institutionen aushungert, übernehmen immer mehr Privatunternehmen die Aufgabe der betrieblichen Gesundheitsförderung (BGF) auf eigene Kosten. Ausserhalb der Politik ist der Bewegungsspielraum weit grösser. Die neue Unternehmensstrategie will Krankheiten, Unfälle und schädlichen Stress am Arbeitsplatz vermeiden, Gesundheitspotentiale stärken und das Wohlbefinden verbessern. Ein landesweites Netzwerk ist, wie auch die SUVA, mit dem europäischen Partner für den Erfahrungs- und Wissensaustausch liiert. Unternehmerische Aktivitäten zur Förderung der BGF sollen ab diesem Jahr mit einem Soziallabel ausgezeichnet werden. Staatliche Massnahmen zur Unfallverhütung haben eine lange Tradition und Rechtsgrundlage, sie haben den Weg zur privaten Initiative geebnet. Die neue Strategie kombiniert ökonomische, technologische, soziale, ökologische und ethische Ansätze mit den alten Zielen der Wirtschaftlichkeit, Produktivität und Rentabilität. Schrittmacher sind vor allem Grossunternehmen, während kleinen und mittleren Betrieben die Zeit, die Kenntnisse und die finanziellen Mittel häufig fehlen. Aus Sicht der Geschäftsleitungen der Betriebe mit Erfahrung überwiegen die Vorteile, wie Senkung der Fehlzeiten und Fluktuation, Erhöhung der Produktivität, Verbesserung des Arbeitsklimas, weniger Krankheitskosten und Imagegewinn. Einzig der erhöhte Arbeitszeitaufwand und die Administration werden negativ bewertet.

\section{Beispiel Migros Ostschweiz}

Die Ideen Gottlieb Duttweilers wirken auch im Industriegelände zwischen Gossau und St. Gallen nach. In der Betriebszentrale für die 9786 Mitarbeitenden, davon $80 \%$ Frauen, befindet sich auch das Büro für den Bereich Gesundheit und Soziales. Gut eingeführt sind zwei Pfeiler der BGF, das Absenzen- bzw. Anwesenheitsmanagement und die Arbeitssicherheit. Den Angestellten steht gratis eine Sozialberatung zur Verfügung. SUVA und Swica sind die eingespielten Partner auf der Versichererseite. Die Ursachen der rund 1500 Unfälle jährlich werden sorgfältig analysiert. Daraus resultieren Massnahmen, wie bauliche oder organisatorische Anpassungen oder Schutzausrüstungen (z. B. Tragepflicht für Sicherheitsschuhe für bestimmte Mitarbeitergruppen [Mitfinanzierung durch Betrieb]). Weitere Aufgaben sind die Tauglichkeitsprüfungen für Nachtarbeit durch die Hausärzte sowie die freiwilligen Grippeimpfungen durch ein SwicaTeam. Ein wichtiger Teil ist das Management der «Fehlzeiten» sprich Absenzen. Gespräche mit Betroffenen analysieren die Gründe, wie Bedingungen am Arbeitsplatz, auf dem Arbeitsweg oder in der Freizeit. Strukturierte Gesprächsunterlagen für die Vorgesetzten, Präsenzspiegel und Belastungsprofile gehören zum Werkzeug der Personalabteilung. Angestrebt ist eine enge Vernetzung mit den Versicherern und Behandelnden zur möglichst raschen Wiedereingliederung. Je nach individueller Situation eines Mitarbeiters erhält der zuständige Arzt einen massgeschneiderten Vorschlag für (vorübergehende) «Schonarbeit», sprich andere Arbeitstätigkeit. Die Gesundheitsförderung im engeren Sinne ist in der Ostschweiz jetzt im Aufbau. Ausgehend von einem Grundsatzpapier des Genossenschaftsbundes sollen Ernährung, Bewegung und Wohlbefinden gezielt gefördert werden. Bereits vorhandenes Wissen und eigene Betriebsressourcen (z.B. Know-how der Klubschulen) als auch Erfahrungen und Grundlagen aus anderen Migros-Betrieben werden einbezogen. 


\section{Und noch einmal der Staat}

Das Staatssekretariat für Wirtschaft (seco) vertritt die Schweiz im europäischen Netzwerk für betriebliche Gesundheitsförderung, dem 25 Mitgliedstaaten angehören. Stress am Arbeitsplatz ist ein gut dokumentiertes Beispiel, dessen Gesundheitskosten und Fehlzeiten die Seco im Jahr 2000 mit 4,2 Milliarden Franken bezifferte. Die SUVA liefert professionelle Mithilfe mit Informationsmaterial, zum Beispiel mit einem Stressvideo, das in Wien mit dem 1 . Preis ausgezeichnet wurde, aber auch Arbeitshefte, Ausbildungskurse und Beratung. Ein Projekt der «Gesundheitsförderung Schweiz» bietet Entwicklungshilfe mit BGF-Modulen für KMUs ab 50 Mitarbeitern.

Eine vorausschauende betriebliche Gesundheitspolitik ist in der Unternehmenskultur vieler privater und staatlicher Grossbetriebe verankert. Ob bei der Warenhauskette Manor, beim Amt für
Strafvollzug des Kantons Waadt oder bei der Zentralwäscherei Zürich, überall wird enorm viel Aufbau- und Entwicklungsarbeit geleistet. Dabei gibt es kein Patentrezept, jede Förderung ist firmenspezifisch und individuell. Kleine, konkrete Verbesserungen bringen oft mehr als grosse Würfe. Vorbildlich ist das Ineinandergreifen staatlicher und privater Initiativen. Für einmal ergänzen sich Rendite und Steuern optimal in einem Teilbereich des helvetischen Gesundheitswesens.

- «Prävention \& Gesundheitsförderung vor dem Grounding?» Im Spannungsfeld zwischen politischer Vernachlässigung und sozialer Notwendigkeit. Symposium der Vereinigung der kantonalen Beauftragten für Gesundheitsförderung, Gossau, 29. April 2004.

- Interview mit Herrn Markus Blunschi, Bereichsleiter «Gesundheit und Soziales», Migros Ostschweiz. 